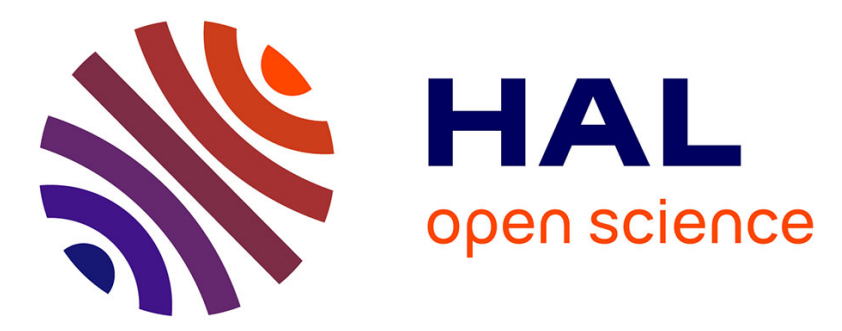

\title{
John Hagan, Who are the Criminals? The Politics of Crime Policy from the Age of Roosevelt to the Age of Reagan
}

\author{
Matthieu de Castelbajac
}

\section{- To cite this version:}

Matthieu de Castelbajac. John Hagan, Who are the Criminals? The Politics of Crime Policy from the Age of Roosevelt to the Age of Reagan. Champ Pénal, 2013, X, http://champpenal.revues.org/8385. hal-00797869

\section{HAL Id: hal-00797869 \\ https://hal.science/hal-00797869}

Submitted on 7 Mar 2013

HAL is a multi-disciplinary open access archive for the deposit and dissemination of scientific research documents, whether they are published or not. The documents may come from teaching and research institutions in France or abroad, or from public or private research centers.
L'archive ouverte pluridisciplinaire HAL, est destinée au dépôt et à la diffusion de documents scientifiques de niveau recherche, publiés ou non, émanant des établissements d'enseignement et de recherche français ou étrangers, des laboratoires publics ou privés. 


\section{Champ pénal/Penal field}

Vol. X | 2013 :

La délinquance en col blanc : études de cas

Lectures

\section{John Hagan, Who are the Criminals? The Politics of Crime Policy from the Age of Roosevelt to the Age of Reagan.}

Princeton, Princeton University Press, 2010, 301 p.

MATThieu de CASTELBAjaC

\section{Texte intégral}

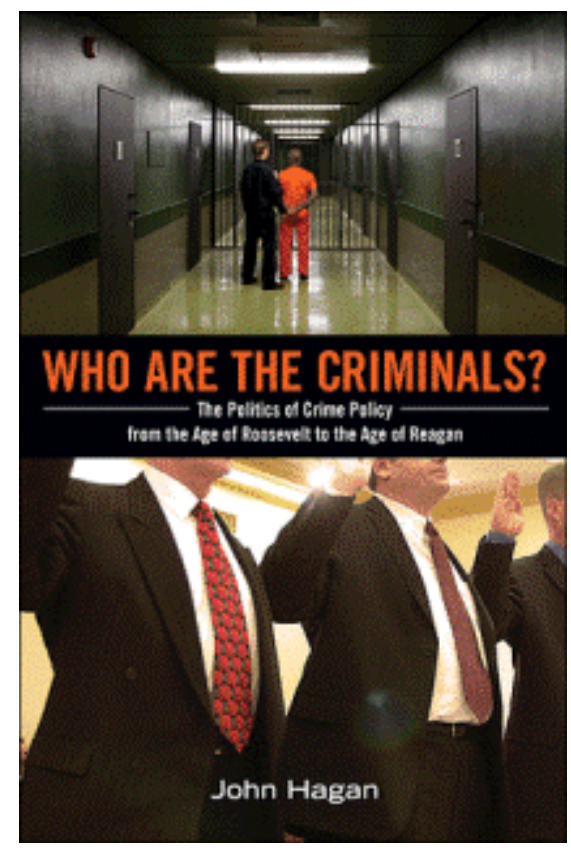

En 1901, Henry George, Jr. ${ }^{1}$ fit paraître un pamphlet intitulé Qui sont les criminels ? Ce court discours se terminait ainsi :

Qui sont les criminels ? Est-ce que ce sont les cambrioleurs, les malheureuses qui font le trottoir et les policiers qui tuent à gages ? Ou est-ce que ce sont ceux qui réduisent les masses à la pauvreté, une pauvreté profonde, incisive, dégradante ?2 
Le titre du dernier ouvrage de John Hagan rend peut-être hommage à ce pamphlet. La question qu'il pose signifie également : «Qui sont les vrais criminels ? ». Hagan se demande en effet, des délinquants en col blanc et des malfrats des rues, qui sont les plus dangereux, et par conséquent, qui la justice devrait punir le plus sévèrement. Hagan constate les fortunes inverses des deux catégories de délinquants, et plaide pour plus d'équilibre.

3 Son plaidoyer repose sur une seconde opposition, introduite dès le sous-titre, entre l'époque de Roosevelt (circa 1933-1973) et l'époque de Reagan (circa 1973-2008). Entre ces deux époques, la différence de traitement entre délinquants en col blanc et malfrats des rues s'est formidablement accrue. Depuis Reagan, les premiers ont prospéré grâce à une politique de laisser-faire, tandis que les seconds ont été condamnés en masse à des peines de prison de longue durée. Pourquoi ? Et avec quelles conséquences ? Hagan soumet ces deux problèmes à des éclairages divers qu'il tire d'une riche connaissance de la littérature criminologique étasunienne.

4 Pour commencer, il exhume le rapport d'une commission présidentielle sur le crime, dont Reagan avait interdit la publication. Ce rapport soulignait que les taux de crime enregistrés par la police avaient baissé, sans que la peur du crime ne diminue. Cette conclusion ne plaisait guère à Reagan et à ses conseillers. Ces derniers voulaient peindre une menace criminelle horrible pour exciter davantage les peurs du public, et mettre un terme aux programmes de réhabilitation des délinquants de droit commun. Selon Hagan, la destruction du rapport secret sur le crime du Président Reagan a coïncidé avec une occasion manquée (...) de redéployer des ressources [monopolisées par] (...) une dépendance de plus en plus grande envers l'emprisonnement (p.18).

5 Dans le chapitre suivant, Hagan propose d'étudier dans un même cadre d'analyse les crimes qui sont habituellement traités comme tels et les crimes qui échappent le plus souvent à la justice pénale, tels que les crimes en col blanc et les crimes d'État. Il ordonne tous les types d'écarts aux normes sur un continuum, selon qu'ils sont jugés plus ou moins graves ; le traitement pénal des différents types de déviance dépend du degré de peur qu'ils suscitent. Or, ce degré de peur est susceptible d'être manipulé : ainsi, bien que les crimes en col blanc soient dangereux, ils sont peu punis, le personnel politique, sous Reagan, ayant réussi à faire croire qu'il était inutile d'en avoir peur.

$6 \quad$ Le troisième et le quatrième chapitres comparent les théories sociologiques du crime de l'époque de Roosevelt et de celle de Reagan. Entre les années 1930 et 1960, trois grands groupes de théories étaient en compétition : le structurofonctionnalisme, l'interactionnisme symbolique et les théories du conflit. Mais ces trois groupes de théorie s'accordaient au moins pour donner une place stratégique aux crimes en col blanc. Dans les années 1970, une innovation théorique et méthodologique a bouleversé le champ : l'analyse longitudinale des carrières criminelles. Les délinquants en col blanc, réputés occasionnels, apparaissaient comme de mauvais candidats pour l'analyse longitudinale ; ils ont donc été moins étudiés que les malfrats des rues, apparemment plus enclins à la récidive.

Dans les chapitres cinq et six, Hagan décrit comment Reagan et ses conseillers ont fait peur au public pour lui vendre l'incarcération en masse des malfrats des rues (qui coûte cher en impôts), tout en faisant croire que la déréglementation des activités bancaires était sans danger, alors qu'elle a par la suite ruiné un nombre extraordinaire de foyers. En déséquilibrant la répartition des peurs du crime, Reagan et ses conseillers ont présidé aux fortunes inverses des deux catégories de criminels. Mais il y a un lien plus surprenant encore entre crimes en col blanc et crimes de rue. La déréglementation du crédit a permis les emprunts frauduleux qui ont financé la construction des prisons dans lesquelles sont venus s'entasser les malfrats des rues !

Le dernier chapitre étudie deux types de crimes d'État : d'une part, la pratique de la torture, par l'armée étasunienne et par la CIA, sous les deux gouvernements de 
George W. Bush ; d'autre part, les cas de « viols d'État » (state rape) répertoriés en Irak, sous Saddam Hussein, et au Soudan, sous Omar al-Bashir. Des agressions sexuelles à grande échelle, directement ou indirectement organisées par la classe dirigeante, ont été employées pour subjuguer des groupes rivaux dans ces deux pays. Mais ces crimes d'État sont très rarement condamnés, alors même qu'ils causent des dommages incommensurables. Bien que Hagan ne prenne pas la peine de justifier cette excursion finale hors des États-Unis, celle-ci poursuit, sur la scène internationale, un des objectifs principaux du livre : dénoncer la façon dont le personnel politique manipule les peurs des citoyens pour opprimer des groupes minoritaires, au moyen d'un harcèlement pénal sélectif.

$9 \quad$ L'épilogue du livre s'aventure sur le terrain de la prédiction. Hagan part du principe que l'élection de Barack Obama a inauguré une nouvelle ère. Oubliant soudain que le nouveau vice-président, J oe Biden, a été l'un des artisans des politiques pénales et carcérales qu'il a dénoncées dans les précédents chapitres (p. 135, 138, 155), Hagan interprète une poignée de signes de bonne volonté, de la part du nouveau gouvernement, comme l'annonce de temps meilleurs. Cet optimisme relève clairement de la pensée magique.

10 Exception faite de son épilogue, le livre de Hagan est passionnant. Il retrace avec clarté et concision l'histoire de la criminologie étasunienne, explique avec pédagogie les montages financiers des délinquants en col blanc, dévoile avec talent des liens inattendus entre crimes de droit commun, crimes financiers et crimes de guerre. Son plaidoyer pour une plus grande vigilance contre les crimes en col blanc et les crimes d'État est assez convaincant. Mais il aurait pu être décisif. En pointant ceux de ses arguments qui mériteraient d'être consolidés, on voudrait contribuer à rendre ce plaidoyer plus éloquent encore.

11 Hagan fait feu de tout bois. Sa connaissance de la littérature criminologique lui fournit des éclairages sur des questions qui sont rarement étudiées ensemble. Cependant, ces éclairages sont disparates et mal reliés entre eux. Hagan prétend les rassembler dans un cadre d'analyse unique, nommé théorie critique des cadrages collectifs. Malheureusement, cette version appauvrit la théorie des cadrages de Goffman, dont elle se dit l'héritière (p. 134, 137). Alors que Goffman distinguait plusieurs façons de cadrer l'expérience d'un public, Hagan ne s'intéresse qu'aux cadrages qui sont le produit d'une manipulation délibérée et malintentionnée. Sans surprise, mais sans plus de précision, Hagan situe ces fourberies au plus haut niveau du pouvoir. À son gré, il étudie quelques cas exemplaires et toujours scandaleux : il dénonce des personnages publics qui ont mis en jeu leur notoriété personnelle ou leur talent unique de communicant pour faire triompher des plans qui, loin de servir le public, ont eu pour lui des conséquences désastreuses.

Cette « dénonciation civique $»^{3}$ est certainement à sa place dans un essai de sociologie critique ; mais elle va un peu vite en besogne. Tout dévoilement s'adresse à un auditoire inconscient de la tromperie dont il a été la dupe. Or, cette lourde hypothèse mériterait d'être étayée. Hagan prête aux déclarations de Reagan et de ses conseillers une efficacité automatique. Tout semble se passer comme si un public dépourvu de sens critique se laissait complaisamment suggestionner. Est-ce bien le cas ? Une étude récente a montré que non : contrairement à une légende sociologique tenace, les sondages des années 1960 n'ont jamais enregistré que des scores très faibles de préoccupation pour le crime. Même au moment des émeutes, la plupart des sondés faisaient la part des choses entre criminels et émeutiers. En bref, l'opinion publique n'a pas cédé à cette époque aux alarmes des tribuns conservateurs. Mais la presse et le personnel politique ont fait comme si c'était le cas ${ }^{4}$.

13 Cet exemple tend à prouver que le personnel politique réussit mieux à scotomiser l'opinion publique qu'à la manipuler. Hagan lui-même cite bien des faits qui illustrent cette possibilité, à commencer par la dissimulation du rapport présidentiel de 1984. De même, il remarque que Reagan était enclin à s'enfermer dans un monde 
imaginaire (p. 168), et qu'il n'était pas homme à laisser des ambiguïtés factuelles (...) interférer avec le développement des images qu'il confectionnait avec art (p. 27). Enfin, le dernier chapitre fait voir comment le personnel politique, même pris la main dans le sac, s'entête à nier les crimes de guerre que la politique a fomentés. Plutôt qu'une théorie de la manipulation de l'opinion, Hagan aurait donc plutôt besoin d'une théorie qui explique la cohérence idéologique des autorités aussi bien que leurs efforts pour censurer ou assimiler toute information gênante ${ }^{5}$.

La préoccupation pour le crime n'ayant pas réellement grimpé au cours des années 1960, on peut se demander si la périodisation que propose Hagan n'est pas faussée. Plus précisément, on peut se demander si le découpage entre l'époque de Roosevelt et l'époque de Reagan ne produit pas une illusion d'optique. À tout prendre, les deux époques sont-elles si différentes ? Hagan soutient que la première aurait accordé une attention plus équilibrée aux crimes de rue et aux crimes en col blanc. Mais cette affirmation se fonde sur deux arguments discutables : le succès du livre White Collar Crime de Sutherland et celui des enquêtes de délinquance auto-dévoilée. Le problème est que Sutherland avait conscience du caractère hétérodoxe et provocateur de son livre, qui a d'ailleurs rallié contre lui les critiques et le scepticisme de nombreux criminologues de son époque. Quant aux enquêtes de délinquance auto-dévoilées, elles ne portaient pas sur les crimes en col blanc, mais sur la question de savoir si les classes aisées étaient réellement moins enclines aux crimes de droit commun que les autres classes.

Ensuite, est-il vrai qu'à partir des années 1970, le débat criminologique s'est détourné des délinquants en col blanc ? Cette impression tient pour une bonne part aux auteurs qu'a sélectionnés Hagan : ils représentent tout au plus son anthologie personnelle, et non un échantillon raisonné. L'impression d'une éclipse des criminels en col blanc aurait sans doute été moins forte si Hagan avait cité, par exemple, les importantes recherches publiées par Albert Biderman et Albert Reiss à ce sujet en $1981^{6}$.

Cette périodisation pâtit encore de son caractère impressionniste. L'époque de Roosevelt et celle de Reagan ne recouvrent pas les mandatures présidentielles du premier et du second ; ce sont deux périodes culturelles plus étendues mais aussi plus floues. Hagan ne dit pas précisément ce qui vaut aux deux présidents l'honneur d'en être les éponymes. De plus, séparant ces deux époques tantôt en 1968 (p. 137 et p. 257), tantôt en 1973 (p. 2 et p. 69), il ne dit pas pourquoi il balance entre ces deux dates. On lui accordera de bon gré que ces bornes approximatives ont seulement une valeur heuristique ; on peut néanmoins supposer qu'elles n'ont pas été arrêtées au hasard. Manifestement, 1968 marque une année politique : Reagan échoue à devenir le candidat républicain à l'élection présidentielle (p. 149), mais il inspire au candidat Nixon le thème de la « Loi et de l'Ordre »; pendant sa campagne, Nixon prononce pas moins de dix-sept discours sur ce sujet (p. 23 et p. 150) ; quand Martin Luther King est assassiné, Washington D.C. est agitée par des émeutes populaires, dont il est question dans une aneodote probablement mythique concernant Reagan rapportée par un de ses conseillers (p. 5) ; enfin, cette année-là, Grant Park est le théâtre d'une importante manifestation contre la guerre du Vietnam (p. 257). Ces événements sont plus ou moins liés entre eux, mais ils sont tous politiques. Au contraire, 1973 est riche en événements théoriques. Blumstein et Cohen, d'un côté, Taylor, Walton et Young, de l'autre, publient des manifestes qui rompent avec les théories classiques du crime. Si Hagan hésite entre 1968 et 1973, c'est donc que les deux séries temporelles ne se chevauchent pas exactement. Il y a comme un cultural lag entre la recherche et la politique.

17 Ce point n'est pas explicité, or il est capital. Hagan suggère que la recherche criminologique est davantage déterminée par la politique que l'inverse. Si les politiciens invoquent régulièrement des travaux de criminologues pour justifier leurs décisions, Hagan ne manque jamais une occasion de souligner qu'il s'agit de 
récupérations peu fidèles (par exemple p. 74). Selon lui, la plupart de ces décisions émanent en réalité de discussions informelles entre politiciens et informateurs plus ou moins bien renseignés (par ex. p. 12-13, p. 154). Au contraire, les criminologues dépendent matériellement de la politique ; ils vivent des financements publics (p. 25, 101, 104) et sont à la merci des pressions du pouvoir. Un exemple extrême est la fermeture forcée du centre de recherches criminologiques de Berkeley en 1976 (p. 100-103). Cette relation inégale contredit le «premier argument » du livre, suivant lequel une interaction permanente entre débat politique et idées [savantes] détermine les politiques criminelles en Amérique (p. 2). La contradiction vient de ce que Hagan réduit la science à une productrice d'idées. Or, si le personnel politique n'est pas le destinataire privilégié des idées scientifiques, il est en échange grand consommateur des productions techniques de la science. Hagan aurait eu intérêt à étudier l'utilisation politique des enquêtes sur les victimes, des modélisations du système judiciaire et des nombreux instruments de prévision que la recherche a confectionnés au cours de la période qu'il étudie. De cette façon, le rapport entre science et politique aurait sans doute paru moins inégal ${ }^{7}$.

Pour finir, il convient de discuter la philosophie pénale de Hagan. Cette philosophie roule sur une définition du crime assez peu objective. Hagan définit le crime par la peur. Ce critère appelle contre lui toutes les objections que Durkheim a levées il y a plus d'un siècle : tout ce qui est dangereux n'est pas criminel ; tout ce qui est criminel n'est pas objectivement dangereux ; et quand bien même on ajoute que certains actes sont interdits parce que, sous l'effet d'une illusion, ils semblent nuisibles, on aboutit à un truisme. C'est comme si on disait que la société traite certains actes comme des dangers à éviter parce qu'elle les croit dangereux ${ }^{8}$.

En outre, cette définition n'aide guère à reconnaître les crimes dont on aurait raison d'avoir peur, et ceux dont on a peur de façon irrationnelle. Après tout, pourquoi faudrait-il avoir moins peur des faits divers montés en épingle par la presse et des tribuns alarmistes ? Et pourquoi faudrait-il montrer plus de crainte pour les crimes en col blanc qu'on ne l'a fait jusqu'à présent ? On accordera volontiers à Hagan que les crimes en col blanc causent de plus grands dommages au plus grand nombre (p. 2). Au contraire, les crimes violents se concentrent sur une minorité, et sont le plus souvent de faible gravité, comme l'ont révélé les enquêtes sur les victimes ${ }^{9}$. Manifestement, le critère suivant lequel Hagan hiérarchise les deux catégories de crime est une variation du principe d'utilité : c'est le rapport entre la quantité de mal et le nombre de victimes. Or, comme le remarquait déjà Durkheim, tout bon que soit ce principe quantitatif, ce n'est malheureusement pas lui qui organise le droit pénal des sociétés modernes : le meurtre d'un individu quelconque est condamné ; une crise économique qui en jette des millions dans la ruine, non. $\mathrm{Si}$ on reprend l'hypothèse durkheimienne suivant laquelle le droit pénal d'une société symbolise sa conscience morale, alors force est de constater que les États-Unis, comme l'ensemble des sociétés modernes, accordent une plus grande importance aux crimes touchant à la personne et aux biens de l'individu qu'aux crimes affectant la collectivité toute entière. On peut le déplorer, mais c'est un fait. La généralité de ce fait affaiblit la thèse d'un particularisme criminologique des États-Unis, que défend Hagan (p. 57-60). Elle n'encourage pas non plus à penser, comme Hagan (p. 211-212), qu'il suffirait d'opposer au discours sécuritaire des quarante dernières années un contre-discours sociologique pour changer la donne. La sociologie est probablement plus utile quand elle s'efforce de rendre visible, d'objectiver et de quantifier les crimes sur lesquels la justice ferme les yeux.

\section{Notes}

1 Il s'agit du fils de l'économiste du même nom. En plus de ses activités littéraires, Henry, Jr. fut représentant de New York au Congrès des États-Unis de 1911 à 1915. 
2 Who are the criminals? Are they the housebreakers, the unfortunate women who walk the streets and the police officials who take blood-money? Or are they those who rob the masses of the people into poverty - deep, biting, degrading poverty? (Henry George, J r., 1901, Who are the criminals?, New York, The Abbey Press, p. 23).

3 Au sens de Luc Boltanski, Laurent Thévenot, 1991, De la J ustification. Les économies de la grandeur, Paris, Gallimard.

4 Dennis Loo, Ruth-Ellen Grimes, 2004, Polls, Politics, and Crime: The "Law and Order" Issue of the 1960s, Western Criminology Review, 5, 1, 50-67.

5 Par exemple J ohn Levi Martin, 2002, Power, authority, and the constraint of belief systems, American J ournal of Sociology, 107, 4, 861-904.

6 Albert Biderman, Albert Reiss, 1981, Data sources on white-collar law-breaking, Washington, D.C., U.S. Dept. of J ustice.

7 Comme le montre la remarquable thèse de Bilel Benbouzid, 2011, La prévention situationnelle : genèse et développement d'une science pratique, Lyon, Université Lumière Lyon 2.

8 Émile Durkheim, 1991[1893], De la division du travail social, Paris, PUF, p. 37-38.

9 Par exemple Albert Reiss, 1981, Marshalling crime statistics, Annals of the American Academy of Political and Social Science, 453, 222-236.

\section{Pour citer cet article}

Référence électronique

Matthieu de Castelbajac, « John Hagan, Who are the Criminals? The Politics of Crime Policy from the Age of Roosevelt to the Age of Reagan. ", Champ pénal/Penal field [En ligne], Vol. X | 2013, mis en ligne le 06 mars 2013, Consulté le 07 mars 2013. URL :

http://champpenal.revues.org/8385

\section{Auteur}

Matthieu de Castelbajac

Doctorant à l'Université de Versailles Saint-Quentin, rattaché au CESDIP (UMR 8183).

Contact : castelbajac@cesdip.fr

\section{Droits d'auteur}

(C) Champ pénal 